\title{
Influence of feed/inoculum ratios and waste cooking oil content on the
} mesophilic anaerobic digestion of food waste

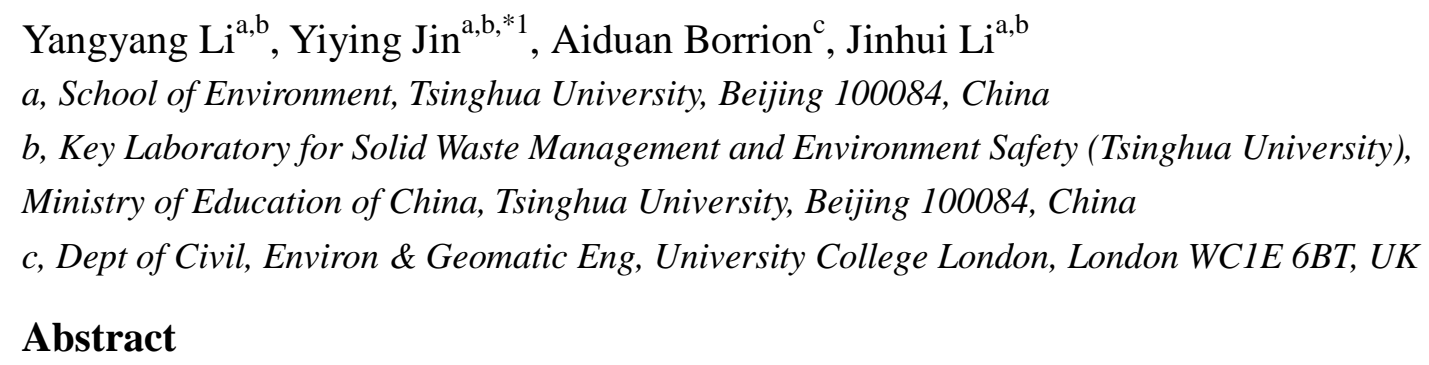

Information on the anaerobic digestion (AD) of food waste (FW) with different waste cooking oil contents is limited in terms of the effect of the initial substrate concentrations. In this work, batch tests were performed to evaluate the combined effects of waste cooking oil content (33-53\%) and feed/inoculum (F/I) ratios (0.5-1.2) on biogas/methane yield, process stability parameters and organics reduction during the FW AD. Both waste cooking oil and the inoculation ratios were found to affect digestion parameters during the $\mathrm{AD}$ process start-up and the $\mathrm{F} / \mathrm{I}$ ratio was the predominant factor affecting AD after the start-up phase. The possible inhibition due to acidification caused by volatile fatty acids accumulation, low $\mathrm{pH}$ values and long-chain fatty acids was reversible. The characteristics of the final digestate indicated a stable anaerobic system, whereas samples with $\mathrm{F} / \mathrm{I}$ ratios ranging from 0.8 -1.2 display higher propionic and valeric acid contents and high amounts of total ammonia nitrogen and free ammonia nitrogen. Overall, F/I ratios higher than 0.70 caused inhibition and resulted in low biogas/methane yields from the FW.

\section{Keywords}

\footnotetext{
* Corresponding author. Address: School of Environment, Tsinghua University, Beijing, China. Tel.: +861062794352; Fax: +861062797618. 
24 Anaerobic digestion of food waste; Biogas; Waste cooking oil; Feed/inoculum ratios;

25 Digestate

26 Abbreviations

27 FW: Food Waste

28 AD: Anaerobic Digestion

29 F/I: Feed/Inoculum

30 EE: Ether Extract

31 VFA: Volatile Fatty Acids

32 LCFA: Long Chain Fatty Acids

33 SFA: Saturated Fatty Acids

34 MUFA: Monounsaturated Fatty Acids

35 PUFA: Polyunsaturated Fatty Acids

36 RT: Retention Time

37 TS: Total Solid

38 VS: Volatile Solid

39 AMPTS: Automatic Methane Potential Test System

40 TAN: Total Ammonia Nitrogen

41 FAN: Free Ammonia Nitrogen

42 


\section{Introduction}

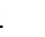

Anaerobic digestion $(\mathrm{AD})$ has been widely applied to reduce the volume of food waste (FW) and to recover energy (e.g., methane) from FW. The content of waste cooking oil in FW may vary from 1\% to 5\% (wet basis) (Li et al., 2016; Nie et al., 2013) due to different eating habits, cooking methods and local cultures (Koch et al., 2015). In addition, waste cooking oil often results in a higher biochemical methane production than carbohydrates and protein (Angelidaki and Sanders, 2004). However, the FW biodegradation processes can be hampered by long-chain fatty acids (LCFAs), which are produced from waste cooking oil and can cause toxicity to microorganisms and biomass adsorption (Chen et al., 2014).

Previous studies have reported various inhibitory concentrations of lipids, including 31-47\% for chemical oxygen demand basis (Cirne et al., 2007) and 65\% for volatile solid (VS) basis (Sun et al., 2014). FW with lipid contents higher than $35 \%$ have been shown to result in $\mathrm{AD}$ processes with longer lag phases and lower first-order degradation constants (Zhang et al., 2017). Studies have also shown that the inhibition caused by LCFAs varies depending on the type of feedstock and is more correlated with the physical characteristics (e.g., specific surface area and size distribution) than the biological characteristics (e.g., inoculum origin, specific acetoclastic methanogenic activity and inoculum adaptation to lipids) of the process (Chen et al., 2008; Hwu et al., 1996). However, these studies were often carried out using either model lipid-rich waste (Cirne et al., 2007) or edible oil (Sun et al., 2014), which have significantly different characteristics from those of waste cooking oil in FW. The 
waste cooking oil existed in FW is of low hygiene quality (Ren et al., 2013; Zhang et al., 2003) and has higher triacylglycerol content (e.g., C14:0, C16:1, C16:0 and C17:0), oleic acid (C18:1) and linoleic acid (C18:2) contents (Zhuang et al., 2013) that are present in the intermediates generated during $\mathrm{AD}$ and are considered to be the main inhibitory factors of LCFAs (Alves et al., 2009). Therefore, investigating the influence of the waste cooking oil ratio on FW digestion performance is necessary. An excessive amount of biomass substrate may lead to the accumulation of total ammonia-nitrogen (TAN) and volatile fatty acids (VFA), resulting in an inhibitory effect on the biogas yield (Fernández et al., 2008; Zhao et al., 2017). Studies have shown that the inhibition caused by LCFAs can be alleviated by increasing the biomass/LCFA ratio using inoculums (Palatsi et al., 2009), and that methane production can decrease or even stop without proper F/I ratios. Additionally, F/I ratios have been reported to affect methane yield mainly with substrates derived from durian shells (Zhao et al., 2017), food and green wastes (Liu et al., 2009), swine slurries (González-Fernández and García-Encina, 2009), wheat straws, whole crop maize, cattle manure, grass, cellulose (Moset et al., 2015) and other organic wastes (Boulanger et al., 2012; Dechrugsa et al., 2013; Fagbohungbe et al., 2015; Haider et al., 2015; Pellera and Gidarakos, 2016). However, considering the potential VFA production and the buffering capacity of the medium using ammonium, each substrate has its own optimum feed/inoculum (F/I) ratio (Lesteur et al., 2010). Moreover, studies examining the combined influence of waste cooking oil and F/I ratios on process stability and biogas/methane productivity in the AD of FW are still lacking. A 
literature review of ether extract (EE) content in FW showed that EE accounts for approximately 6-45\% of the total FW in China (VS basis) (Li et al., 2016; Nie et al., 2013). Since lipid-rich waste is more likely to result in operational problems (Chen et al., 2008; Cirne et al., 2007; Long et al., 2012), the influence of higher waste cooking oil ratios, specifically EE/VS feedstock ratios ranging from $33 \%$ to $53 \%$, were investigated in the present study (Table 1).

This paper aims to investigate the AD characteristics of FW containing different waste cooking oil and F/I ratios. The modified Gompertz model was applied to describe biogas production process and to determine the digestion efficiency, which were then further evaluated to determine how and over which ranges the two ratios affect digestion performance, process kinetics and biodegradability. From this analysis, possible inhibitory effects were discussed, and the optimal waste cooking oil and F/I ratios that increased methane yields were presented.

\section{Materials and Methods}

\subsection{Substrates and inoculum}

\subsubsection{FW}

FW was collected from a school canteen in Beijing, China. Impurities in the collected FW (e.g., big bones, plastics and metals) were manually removed before the FW was macerated into $1-2 \mathrm{~mm}$ particles. The main characteristics of the FW used in the experiments were (average values of three determinations with standard deviations) shown in Table 1. 
(analytically pure, boiling point: $30-60{ }^{\circ} \mathrm{C}$ ) using a rotary evaporator at $60 \mathrm{rpm}$. Then

ratio was characterized by the concentration of the EE in the VS of the FW (EE/VS):

$$
E E / V S=\frac{m_{F W} \times E E_{F W} \%+m_{\text {oil-extracted }}}{m_{F W} \times V S \%+m_{\text {oil-extracted }}} \times 100 \%
$$

113 where $m_{F W}$ is the mass of the initial $\mathrm{FW}, E E_{F W} \%$ is the percent of EE in the 114 initial FW sample, $m_{\text {oil-extracted }}$ is the mass of waste oil added in the FW, which was extracted from FW, and VS\% is the VS content of the initial FW. Table 2 presents the LCFA composition of the waste cooking oil in the FW.

Table 1. Characteristics of the FW and the F/I ratios.

\begin{tabular}{|c|c|c|c|c|c|c|c|}
\hline \multirow{2}{*}{ Items } & \multicolumn{7}{|c|}{ Waste cooking oil ratios (EE/VS) } \\
\hline & $33 \%$ & $36 \%$ & $40 \%$ & $43 \%$ & $46 \%$ & $50 \%$ & $53 \%$ \\
\hline $\mathrm{pH}$ & $4.47 \pm 0.21$ & $4.46 \pm 0.32$ & $4.46 \pm 0.28$ & $4.46 \pm 0.41$ & $4.45 \pm 0.32$ & $4.45 \pm 0.29$ & $4.44 \pm 0.44$ \\
\hline $\mathrm{TS}^{\mathrm{a}, \mathrm{b}}(\%)$ & $15.01 \pm 0.98$ & $15.47 \pm 0.71$ & $16.41 \pm 0.63$ & $17.02 \pm 0.42$ & $17.76 \pm 0.45$ & $18.71 \pm 0.29$ & $19.93 \pm 0.35$ \\
\hline $\mathrm{VS}^{\mathrm{a}, \mathrm{c}}(\%)$ & $14.18 \pm 0.52$ & $15.52 \pm 0.82$ & $18.06 \pm 0.91$ & $19.71 \pm 0.71$ & $21.63 \pm 0.50$ & $23.98 \pm 0.46$ & $26.90 \pm 0.49$ \\
\hline $\operatorname{Protein}^{\mathrm{a}}(\%)$ & $3.58 \pm 0.15$ & $3.56 \pm 0.22$ & $3.53 \pm 0.06$ & $2.17 \pm 0.06$ & $2.15 \pm 0.33$ & $2.12 \pm 0.17$ & $2.09 \pm 0.28$ \\
\hline $\mathrm{F} / \mathrm{I}^{\mathrm{d}}$ & 1.20 & 1.00 & 0.80 & 0.70 & 0.60 & 0.56 & 0.50 \\
\hline
\end{tabular}

${ }^{\mathrm{a}}$ : wet basis; ${ }^{\mathrm{b}}$ : total solid; ${ }^{\mathrm{c}}$ : volatile solid; ${ }^{\mathrm{d}}$ : feed to inoculum ratio

119 Table 2. LCFA composition of the waste cooking oil extracted from the FW (\%).

\begin{tabular}{|c|c|c|c|c|c|}
\hline \multirow{2}{*}{$\mathrm{SFA}^{\mathrm{a}}$} & \multirow{2}{*}{ Content } & \multicolumn{4}{|c|}{ Unsaturated fatty acid } \\
\hline & & MUFA $^{b}$ & Content & PUFA $^{c}$ & Content \\
\hline
\end{tabular}




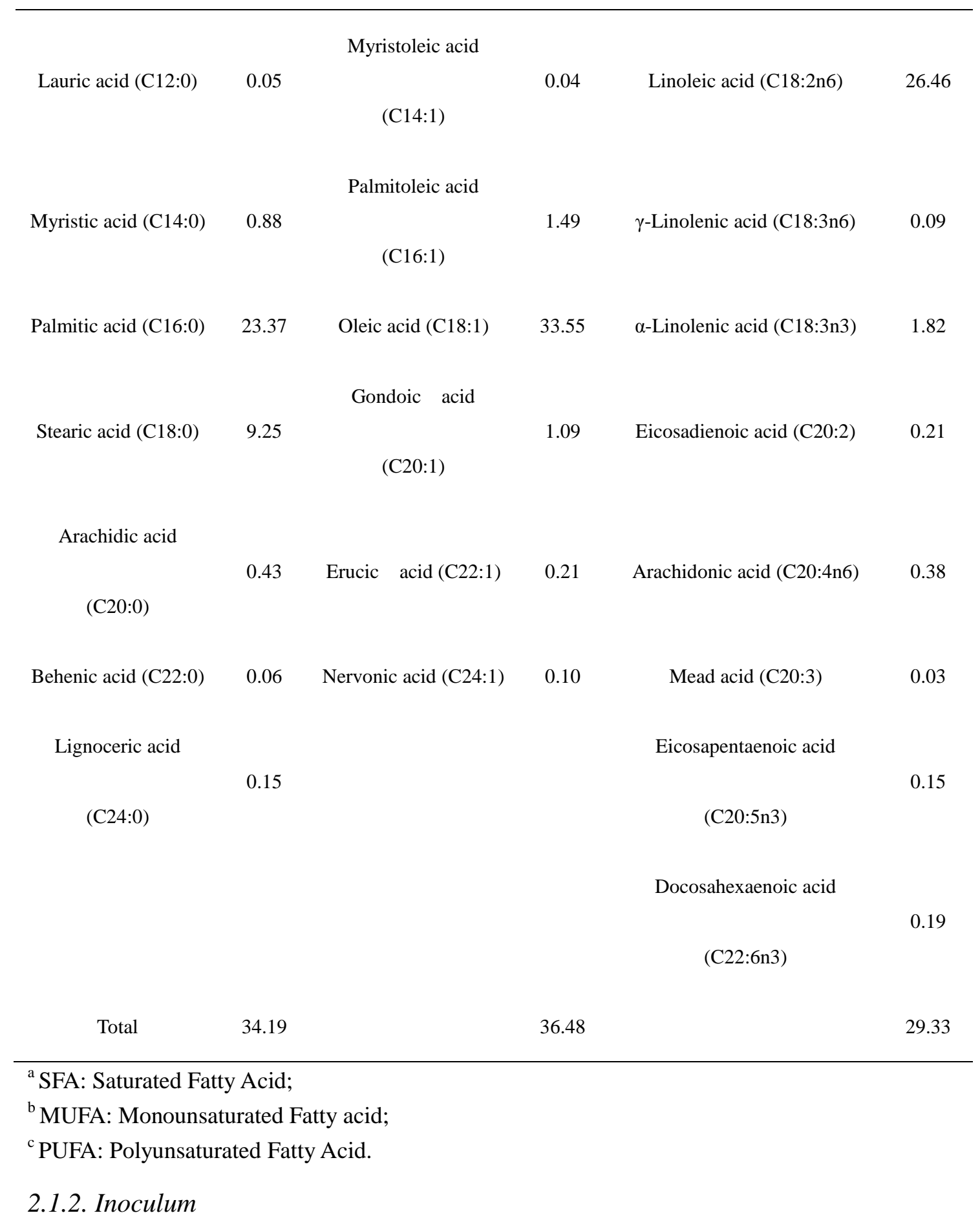

124 Seed sludge was obtained as an inoculum from a steady-operation digester $\left(37^{\circ} \mathrm{C}\right)$

125 at a wastewater treatment plant in Beijing, China. After a two-day gravity

126 sedimentation period, the supernatant was discarded, and the remainder was passed

127 through a 2-mm sieve to remove large particles/grit. The characteristics of the

128 inoculum are shown in Table 3. 
Table 3. Characteristics of the inoculum.

\begin{tabular}{cccccc}
\hline Parameter & $\mathrm{pH}$ & $\mathrm{TS}(\%)$ & $\mathrm{VS}(\%)$ & Ammonia (mg/L) & $\mathrm{C} / \mathrm{N}^{\mathrm{a}}$ \\
\hline Value & 7.34 & $3.65 \%$ & $2.42 \%$ & 1123 & 7.01 \\
\hline a : carbon to nitrogen ratio & & & &
\end{tabular}

\subsection{AD experimental setup}

132

\subsubsection{Determination of the inoculum ratios}

To identify the synergistic impacts of the F/I and EE/VS ratios on FW digestion, we

focused on their interactions with inoculum ratios in digestion experiments on a VS basis (Table. 1). All the F/I ratios as shown in Table 1 were based on a mass of VS basis.

\subsubsection{Batch digestion tests}

Batch tests were conducted in 15 parallel 500 -mL glass bottles at $37{ }^{\circ} \mathrm{C}$ with an automatic methane potential test system II (AMPTS II) that was supplied by

Bioprocess Control (Lund, Sweden). AMPTS II features automatic sample stirring, an acid gas (such as $\mathrm{CO}_{2}$ or $\mathrm{H}_{2} \mathrm{~S}$ ) removal system and a biomethane yield recording system. The system performs fast and accurate on-line measurements of ultra-low biogas and biomethane flow to determine the biogas potential. All the reactors were started simultaneously and used synchronous agitation at the same speeds (160 r/min) and intervals (60 seconds on/off).

The substrates and inoculums were placed into bottles with different F/I ratios. The upper area of each reactor was flushed with nitrogen for at least 1 min to ensure anaerobic conditions and was then quickly sealed. All of the reactors were placed in a 
water bath to maintain the digestion system at a mesophilic temperature $\left(37^{\circ} \mathrm{C}\right)$ for AD. For each test, three samples were examined, and two digesters containing only inocula were incubated to correct for the biogas yield from the inoculum. The biogas yield was calculated by the VS of substrate in the bottle, including FW and waste cooking oil added. The digestion assays were stopped when the daily biogas (or methane) production was less than the $1 \%$ of the total accumulated biogas (or methane).

\subsubsection{Digesters with two volume types}

An AMPTS II system containing 500-mL (total volume) glass bottles (A) was used to measure real-time methane productivity and kinetics, whereas a system with 2-L (total volume) glass bottles $(B)$ was used for sample collection and detection. All of the bottles in both systems were fed with the same samples and inoculums with different F/I ratios (Table 1). To achieve accurate results, collecting samples at the correct times (e.g., the inhibition stage, recovery stage and final stage) is important. Digestion system $A$ was started two days prior to system $B$ to understand how the sample collection time affected the methane yield patterns.

\subsection{Kinetics study}

A kinetics analysis can provide insights into the influences of the F/I and EE/VS ratios on the potential behaviour of organics degradation in the digestion system, such as the lag phase, which is an important factor in determining the AD efficiency. The modified Gompertz model was used to describe the biogas yield potential, the lag phase and the maximum biogas production rate: 


$$
M=P \times \exp \left\{-\exp \left[R_{\max } \times e \times(\lambda-t) / P+1\right]\right\}
$$

172 where $M$ is the cumulative biogas production $(\mathrm{mL} / \mathrm{g} \mathrm{VS})$ at the digestion time $t, P$ is the biogas production potential ( $\mathrm{mL} / \mathrm{g} \mathrm{VS}$ ), $R_{\max }$ is the maximum biogas production rate $(\mathrm{mL} / \mathrm{g} \mathrm{VS} \cdot \mathrm{h}), \lambda$ is the lag time $(\mathrm{h}), t$ is the retention time $(\mathrm{h})$, and $e$ is the exponential constant 2.7183 .

\subsection{Statistical analysis}

\subsubsection{Pearson correlation analysis}

The Pearson correlation $(p<0.05)$ was determined to describe significant relationships between the above parameters, using the IBM SPSS Statistics 20 software (Table A1).

\subsubsection{Second-order polynomial model analysis}


193

194

195

196

197

198

199

200

201

202

203

204

205

206

207

208

209

210

211

212

213

214

\subsection{Analytical methods}

The $\mathrm{pH}$ was measured using a $\mathrm{pH}$ metre (FE20, Mettler, Switzerland). The TS and VS were determined according to the standard methods of the American Public Health Association (APHA, 1992). VFAs were measured using an Agilent Gas

Chromatograph (Agilent GC-7890A, California, USA) equipped with a flame ionization detector. The concentrations of protein and EE were determined with the Kjeldahl method and a Soxhlet device, respectively (Naumann and Bassler, 1993). The concentrations of TAN and free ammonia nitrogen (FAN) were determined as previously reported (Siles et al., 2010). LCFAs were determined in accordance with the method of (Palatsi et al., 2009).

\section{Results and Discussion}

\subsection{Biogas production and methane content}

\subsubsection{Characteristics of biogas production and methane content}

(1) Cumulative biogas yield

The biogas yield ranged from 524 to $1035 \mathrm{~mL} / \mathrm{g}$ VS after 150 days of digestion, whereas the methane percentage of the biogas varied between $67 \%$ and $75 \%$ (Fig. 1A). Lipids had the highest biochemical methane potential of the FW contents (Angelidaki and Sanders, 2004), therefore, FW with higher EE/VS and lower F/I ratios resulted in higher biogas yields $(p<0.05)$ and higher methane contents $(p<0.01)$. The highest yield was achieved from $\mathrm{FW}$ with an EE/VS ratio of $43 \%$ and an F/I ratio of 0.70 and FW with an EE/VS ratio of 46\% and a F/I ratio of 0.60, ranging from 1015 to 1035 $\mathrm{mL} / \mathrm{g}$ VS. These two treatments were $8.44-93.70 \%$ higher than the other treatments. 

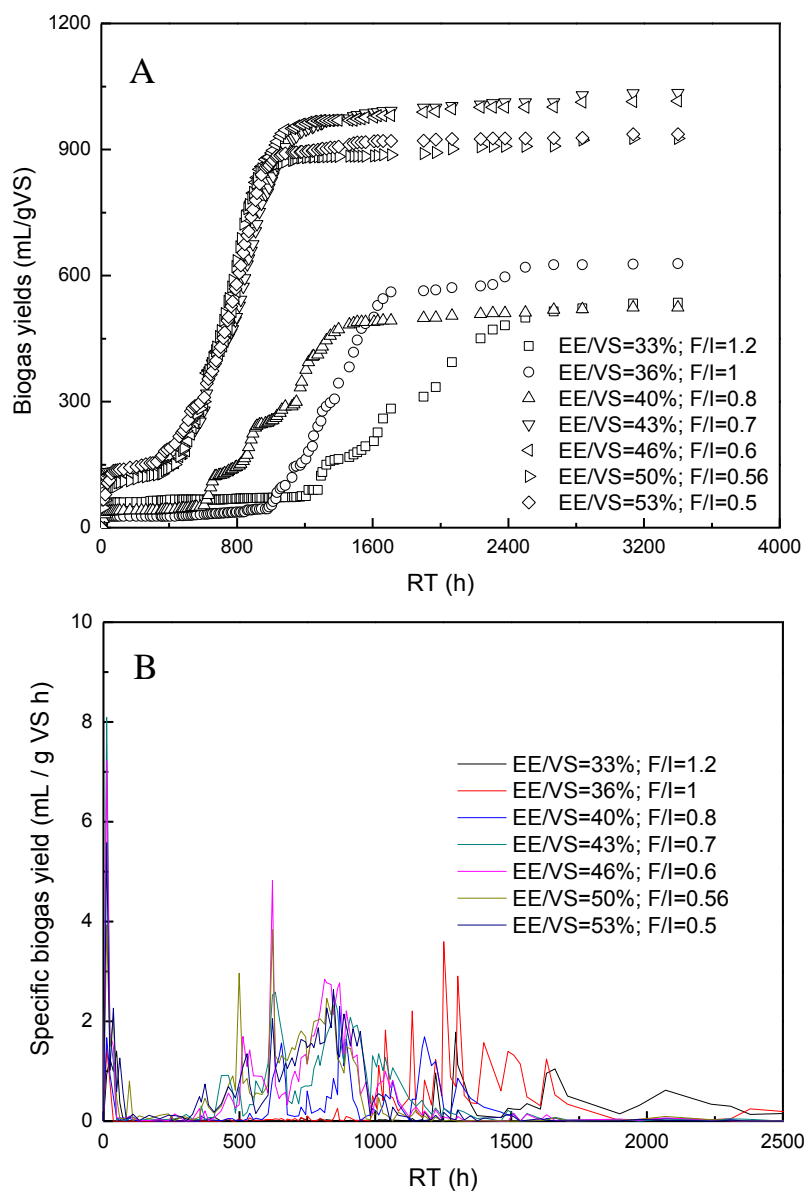

216 Fig. 1. Effect of EE/VS and F/I ratios on the cumulative $\left(\mathrm{A}^{*}\right)$ and specific (B) biogas

217 yield with increased retention time (RT).

$218 \quad$ *: average biogas yields.

219 (2) Specific biogas yield rate

220 The degradation of the FW exhibited intense production during the first $30-40 \mathrm{~h}$,

221 followed by broader and smaller peaks (Fig. 1B). In the sample with F/I and EE/VS

222 ratios of 0.7 and $43 \%$, respectively, relatively intense methane production occurred,

223 with the peak value achieved within the first $12 \mathrm{~h}(8 \mathrm{~mL} / \mathrm{g} \mathrm{VS} \mathrm{h})$.

224 Two main peaks were obtained from the samples. The first peak was of shorter duration and larger maximum height than the second peak. The occurrence of these 
peaks was due to the degradation of easily degradable organics and macromolecular insoluble materials (such as proteins and lipids). The different peak patterns likely result from different FW organic compositions, particularly lipid ratios (EE/VS). The first peak may be due to the biodegradation of carbohydrates, which are converted more rapidly than lipids and proteins (Mata-Alvarez et al., 2000). The second peak may be attributed to the combined degradation of proteins and lipids as well as any remaining carbohydrate.

Table 4 summarizes the biogas yield, starting/ending times and durations of these two peaks. Lower biogas/biomethane yields and $\mathrm{pH}$ values (ranging from 5.14 to 5.55) were achieved at the end of the first stage, and the second stage was characterized by higher biogas production (408-786 mL/g VS) and a longer retention time (767-1465 h). Biogas was mainly produced in the second stage, which accounted for $73-83 \%$ of the total production, and the retention time for the second stage was 1.35-3.97 times as long as that of the first stage. The lower biogas yield in the first stage was probably influenced by decreased $\mathrm{pH}$, which is not compatible with normal methanogens growth (Zhang et al., 2014). Moreover, higher F/I and lower EE/VS ratios resulted in longer retention times for the two stages, and a significant correlation between these parameters $(p<0.01)$ was achieved, while only the $\mathrm{F} / \mathrm{I}$ ratio significantly correlated with the biogas yield in the second stage (Table A1). These findings are likely due to both the F/I and EE/VS ratios being the main factors that affect AD start-up (first stage), which is associated with the initial production of fermentative products. After the start-up phase (second stage), the F/I ratios became the predominant factor 
248 affecting the bio-transform of initial fermentation products by the acetogenic and

249 methanogenic communities.

250 Table 4. Characteristics of biogas production from the FW.

\begin{tabular}{llllllll}
\hline EE/VS & $33 \%$ & $36 \%$ & $40 \%$ & $43 \%$ & $46 \%$ & $50 \%$ & $53 \%$ \\
\hline F/I & 1.20 & 1.00 & 0.80 & 0.70 & 0.60 & 0.56 & 0.50 \\
\hline
\end{tabular}

1. The first stage of biogas production

\begin{tabular}{|c|c|c|c|c|c|c|c|}
\hline Starting time (h) & 0 & 0 & 0 & 0 & 0 & 0 & 0 \\
\hline Ending time $(\mathrm{h})$ & 1085 & 967 & 552 & 304 & 407 & 360 & 262 \\
\hline Duration (h) & 1085 & 967 & 552 & 304 & 407 & 360 & 262 \\
\hline Biogas yield (mL/g VS) & 72 & 44 & 49 & 145 & 148 & 129 & 151 \\
\hline Percentage in total ${ }^{\mathrm{a}}(\%)$ & 13 & 7 & 9 & 14 & 15 & 14 & 16 \\
\hline $\mathrm{pH}$ at the ending time & 5.48 & 5.55 & 5.14 & 5.24 & 5.47 & 5.46 & 5.55 \\
\hline
\end{tabular}

2. The second stage of biogas production

\begin{tabular}{|c|c|c|c|c|c|c|c|}
\hline Starting time $(\mathrm{h})$ & 1085 & 967 & 552 & 304 & 407 & 360 & 262 \\
\hline Ending time $\left(\mathrm{t}_{90}\right)^{\mathrm{b}}(\mathrm{h})$ & 2550 & 2308 & 1708 & 1510 & 1222 & 1173 & 1029 \\
\hline Duration (h) & 1465 & 1341 & 1156 & 1206 & 815 & 813 & 767 \\
\hline Biogas yield (mL/g VS) & 408 & 520 & 419 & 786 & 765 & 704 & 686 \\
\hline Percentage in total ${ }^{\mathrm{a}}(\%)$ & 76 & 83 & 80 & 76 & 75 & 76 & 73 \\
\hline $\mathrm{pH}$ at the ending time & 7.11 & 7.14 & 6.99 & 7.25 & 7.12 & 7.20 & 7.22 \\
\hline \multicolumn{8}{|l|}{ 3. Total AD process } \\
\hline $\mathrm{V}_{90}{ }^{\mathrm{c}}(\mathrm{mL} / \mathrm{g} \mathrm{VS})$ & 480 & 564 & 468 & 931 & 912 & 833 & 837 \\
\hline Total biogas yield (mL/g VS) & 535 & 628 & 524 & 1035 & 1015 & 926 & 936 \\
\hline Methane content (\%) & 67 & 67 & 72 & 73 & 72 & 74 & 75 \\
\hline$T B Y^{\mathrm{d}}(\mathrm{mL} / \mathrm{g} \mathrm{VS})$ & 1129 & 1130 & 1133 & 1098 & 1110 & 1128 & 1140 \\
\hline$T M B Y^{e} / T B Y(\%)$ & 47 & 56 & 46 & 94 & 91 & 82 & 82 \\
\hline \multicolumn{8}{|c|}{${ }^{a}$ Ratios of the biogas yield in this stage to total biogas yield in the whole digestion process. } \\
\hline
\end{tabular}


${ }^{\mathrm{d}}$ Theoretical biogas yield (TBY) was calculated according to the reference (Labatut et al., 2011).

${ }^{\mathrm{e}}$ TMBY: Total measured biogas yield.

256 The ratios of measured biogas yield to theoretical biogas yield varied from $47 \%$ to

$25794 \%$, indicating a lower biogas conversion efficiency for higher F/I ratios. The highest 258 yield ratio (94\%) was achieved with F/I and EE/VS ratios of 0.70 and $43 \%$,

259 respectively, whereas the highest biogas yield was achieved with a methane

260 proportion of $73 \%$ in the biogas (Table 4). These results indicated that the F/I and

261 EE/VS ratios are essential factors that influence the biogas yield, methane content and digestion time during the batch $\mathrm{AD}$ of FW.

\subsubsection{Kinetics evaluation}

Table 5 summarizes the biogas production potential $(P)$, maximum biogas yield rate $\left(R_{m}\right)$ and lag time $(\lambda)$ according to a modified Gompertz model. The high determination coefficients $\left(R^{2}, 0.9173-0.9822\right)$ and high $P$ to total measured biogas

267 yield ratios (94-103\%) for all of the runs showed that the experimental biogas production data could be well simulated using this model. 
Table 5. Results of the kinetics parameters from the modified Gompertz model.

\begin{tabular}{ccccccccc}
\hline EE/VS & F/I & $P$ & $R_{m}$ & $\lambda$ & & $P / T M B Y^{a}$ & $P / T B Y^{b}$ & $\lambda / t_{1}{ }^{c}$ \\
& & $(\mathrm{~mL} / \mathrm{g} \mathrm{VS})$ & $(\mathrm{mL} / \mathrm{g} \mathrm{VS} \mathrm{h})$ & $(\mathrm{h})$ & & $(\%)$ & $(\%)$ & $(\%)$ \\
\hline $33 \%$ & 1.20 & 517 & 0.29 & 872 & 0.9173 & 103 & 46 & 80 \\
$36 \%$ & 1.00 & 615 & 0.79 & 963 & 0.9822 & 102 & 54 & 100 \\
$40 \%$ & 0.80 & 537 & 0.61 & 561 & 0.9796 & 98 & 47 & 102 \\
$43 \%$ & 0.70 & 1063 & 1.19 & 267 & 0.9644 & 97 & 97 & 88 \\
$46 \%$ & 0.60 & 1075 & 1.46 & 347 & 0.9633 & 94 & 97 & 85 \\
$50 \%$ & 0.56 & 905 & 2.07 & 430 & 0.9760 & 102 & 80 & 119 \\
$53 \%$ & 0.50 & 953 & 1.52 & 342 & 0.9611 & 98 & 84 & 131 \\
\hline
\end{tabular}

${ }^{\text {a }} T M B Y$ : Total measured biogas yield.

$271{ }^{\mathrm{b}} T B Y$ : Theoretical biogas yield (TBY) was calculated according to the reference (Labatut et al., 2011).

$272{ }^{c} t_{1}$ : Ending time of the first stage of biogas production in Table 4.

273 Samples with lower EE/VS $(p<0.05)$ and higher F/I ratios $(p<0.01)$ had longer

274 lag times, especially samples with F/I ratios higher than 0.80 (ranging from 561 to

$275963 \mathrm{~h})$, even if the waste cooking oil content was relatively low (33-40\%). These

276 findings may be due to diffusion limitations imposed by the lipid layer surrounding

277 the bacterial cells at high organic loadings, the slow degradation of lipids or the

278 possible inhibition of methanogenic activity by high LCFA concentration (Chen et al.,

279 2014; Long et al., 2012). The F/I ratio could drive the start-up phase of the anaerobic

280 digester, likely due to the degradation of initial hydrolysis products by the

281 methanogenic consortia. Additionally, decreasing F/I ratios may dilute the inhibitory

282 or toxic compounds produced from LCFAs, which was also confirmed by the longer 
duration of the first stage of biogas production (Table 4).

Samples at EE/VS and F/I ratios of $43 \%$ and 0.70 , respectively, had the shortest lag times and achieved high biogas production potential (1063 mL/g VS). Samples with lower EE/VS and higher F/I ratios exhibited lower biogas yields and lower ratios of $P$ to total measured biogas yield (Table 5). These low values may be due to acidification during the first digestion stage. Moreover, further increases in the EE/VS ratio and decreases in the F/I ratio resulted in longer lag times and lower biogas production. Thus, feedstock with higher EE/VS ratios (46-53\%) requires lower F/I ratios than those currently used to minimize and overcome inhibition.

The $\lambda$ values exhibited significant positive correlations $(p<0.01)$ with the $t_{90}$ values (Table A1), and assays with a longer $\lambda$ values had lower biogas yields and methane content. Additionally, samples with high F/I ratios had significantly $(p<0.01)$ decreased $R_{m}$ values (0.29-2.07 $\mathrm{mL} /(\mathrm{g} \mathrm{VS} \mathrm{h})$ ) due to their long lag phases, whereas the EE/VS ratios exhibited positive effects $(p<0.01)$. Therefore, a high F/I ratio increases the duration of the adaptation phase. This result indicated that a good F/I ratio (i.e., less than 0.7 ) is beneficial for microorganism growth and biogas production, and high inoculum concentrations may shorten the digestion time.

In addition, higher $R_{m}$ values resulted in shorter lag phases, lower $t_{90}$ values, and higher biogas production. High methane conversion efficiency for EE compared with carbohydrates and proteins may lead to high biogas yield rates, implying that a relatively large EE/VS ratio is good for the $\mathrm{AD}$ system. The $R_{m}$ values have a positive correlation $(p<0.05)$ with the $P$ values $(517-1075 \mathrm{~mL} / \mathrm{g} \mathrm{VS})$, whereas the $\lambda$ values 
exhibited negative effects $(p<0.05)$. These relationships likely indicate that high biogas conversion efficiency and quick adaptation to a new substrate are essential factors for an inoculum that influence the ultimate biogas yield in the batch $\mathrm{AD}$ of FW.

\subsection{Characteristics of the performance parameters and possible inhibition}

\subsubsection{VFAs}

As shown in Table 4, various durations of the first stage of biogas production were achieved due to the differences in the EE/VS and F/I ratios. Since the VFA concentration had a significant influence on the $\mathrm{pH}$ value, it is essential to study variations of the VFA concentration and composition, especially in the first stage from day 11 to 45 (Fig. 2).

The VFA concentration first increased continuously to a peak, and assays with lower EE/VS and relatively higher F/I ratios presented higher peak values, indicating a rapid build-up of VFAs. Because of their high F/I ratios, the quantities of microorganisms were too low to degrade the initial fermentation products in the soluble fraction of these samples. Therefore, more time was needed to decrease their VFA concentrations, corresponding to longer lag phases. Additionally, during the first stage of biogas production, the low $\mathrm{pH}$ values (ranging from 5.1 to 5.6 at the end of the first biogas yield stage) caused by the high VFA concentrations resulted in inhibition of the methane yield process (Table 4), indicating low biogas yields as retention time increased until the end of the first biogas yield stage (Fig. 1A). However, after an initial lag phase (267-963 h), the accumulated VFA concentration 
decreased, resulting in a $\mathrm{pH}$ increase, which caused the biogas production process to start again. These findings imply that the acidification caused by high VFA concentrations and consequent low $\mathrm{pH}$ values in the present study are reversible. This hypothesis was strengthened by the fact that acetogenic and methanogenic communities are much more sensitive to low growth rates compared with fermentative and hydrolytic populations (Niu et al., 2014).

Acetic acid and $n$-butyric acid are the two main components of the total VFAs, accounting for $50-72 \%$ (Fig. 2). Samples with EE/VS ratios ranging from $33 \%$ to 40\% exhibited similar tendencies in their VFA compositions, the main component of which was $n$-butyric acid (varying from $30 \%$ to $60 \%$ ); after day 11, the propionic and valeric acid contents also increased. For samples with EE/VS ratios of 43-53\%, earlier increases in propionic and valeric acid were observed on day 5 , and the concentration of $n$-butyric acid was higher than that of valeric acid after days $25-30$, while propionic acid was higher than acetic acid for samples with EE/VS ratios of 33-40\%. An accumulation of acetic, propionic and butyric acid was observed for the samples with lower EE/VS and high F/I ratios, resulting in lower biogas yields and $\mathrm{pH}$ values. An inhibition of propionic acid degradation was reported by (Raposo et al., 2006) when the acetic acid concentration was greater than $1400 \mathrm{mg} / \mathrm{L}$. However, the present study revealed that biogas production was still inhibited at values lower than $600 \mathrm{mg} / \mathrm{L}$. Additionally, during the first stage, no major variations in the biogas yield were observed after day 3, during which time there was rapid VFA production; notably, the concentration of propionic acid did not exceed the threshold required for 
methanogenic activity inhibition at $900 \mathrm{mg} / \mathrm{L}$, as reported by (Wang et al., 2009). This finding suggested that the levels of VFAs required for inhibition may depend on feedstock compositions, which indicates that it is not feasible to define specific VFA inhibitory levels. Thus, anaerobic digester failure may be due to different operating parameters (e.g., characteristics of feedstock and inoculum).

\section{However, considering the propionic acid to acetic acid ratio range, values greater} than 1.4 indicated impending digester failure, which may serve as a satisfactory indicator for the beginning of organic overloading (Tang et al., 2008). As shown in Fig. 2 (F), the ratios increased to $1.0-1.2$ on day 5 , and higher values and longer durations were achieved for lower EE/VS and higher F/I ratios, implying low biogas production. The values varied from 0.60 to 0.79 at the end of the first stage and were accompanied by the start-up of biogas production. Thus, the low biogas yields in the first stage after day 5 (Fig. 1) may be due to inhibition caused by higher concentration of propionic acid, which is an undesirable intermediate product in the anaerobic process due to its slower metabolism via methanogenesis (i.e., the low conversion rate of propionic acid to acetic acid and $\mathrm{H}_{2} / \mathrm{CO}_{2}$ ) than acetate and butyrate (Zhang et al., 2005). In addition, neither methane content nor biogas yield showed a significant relationship with $\mathrm{pH}$, but both exhibited a significant negative relationship $(p<0.01)$ with the VFA concentration. These findings disagree with a previous research report (Kawai et al., 2014) which found that the methane content demonstrated no significant relationship with the VFA concentration, but had a significant positive relationship with $\mathrm{pH}$. This discrepancy may be due to differences in the substrate 
371 characteristics, especially for the FW with higher EE content in this study. The VFA

372 concentrations and compositions in the batch AD of FW may have been influenced by 373 a synergistic effect of the EE/VS and F/I ratios. 

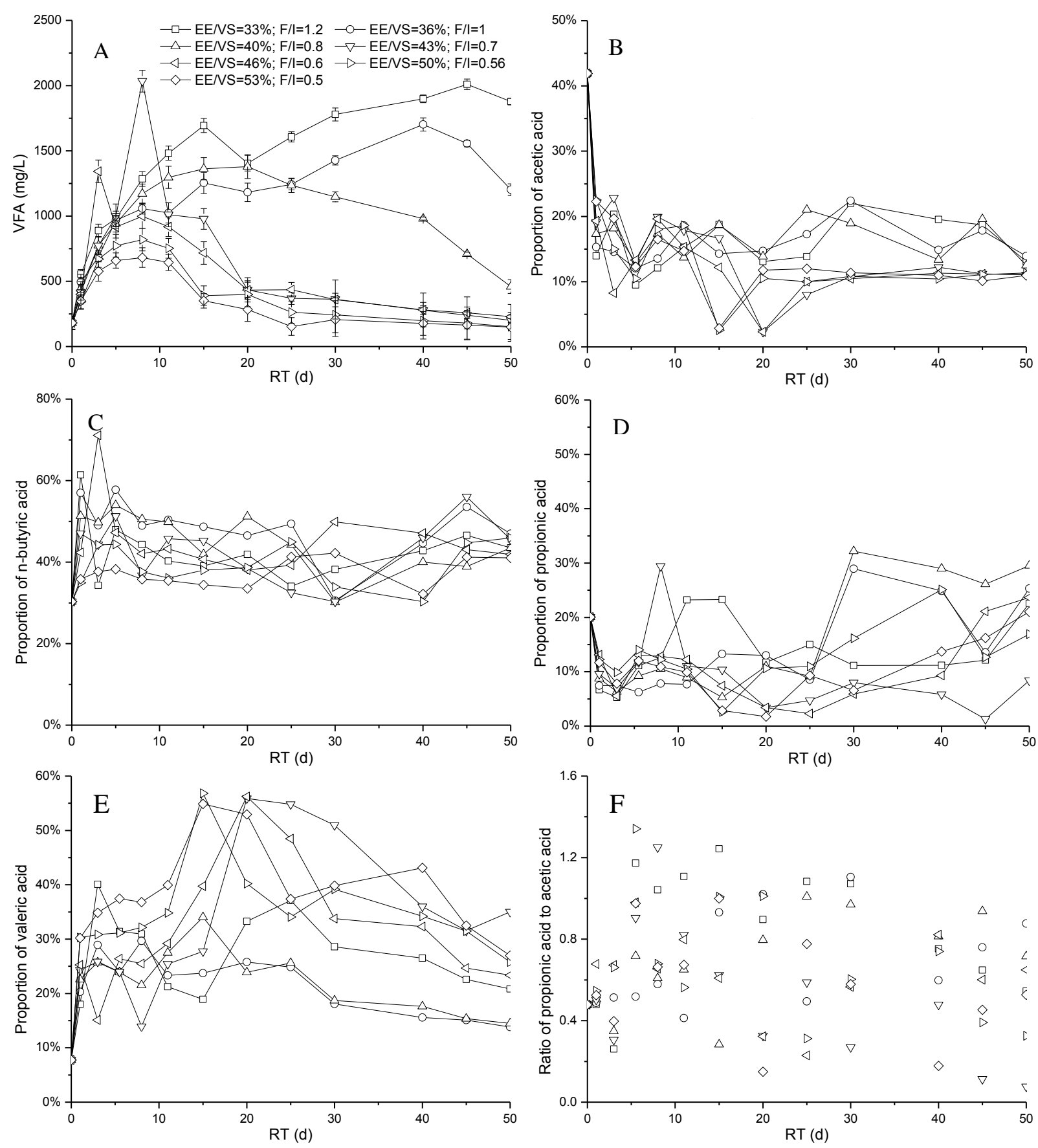

374 Fig. 2. Effects of the EE/VS and F/I ratios on the VFA concentrations (A), VFA compositions (B, C, D and E) and propionic acid to acetic acid ratios (F) with

376 increased retention time (RT). 
当前使用的样式是 [Energy Policy]

当前文档包含的题录共 61 条

有 0 条题录存在必填字段内容缺失的问题

所有题录的数据正常

\subsubsection{LCFAs}

The major fatty acid constituents in the samples used in this study were unsaturated fatty acids, especially oleic acid (C18:1) and linoleic acid (C18:2), whereas palmitic acid (C16:0) was the main saturated fatty acid (Table 2). High concentrations of waste cooking oil in the initial substrate resulted in the high contents of these three constituents. According to the biogas yield pattern (Fig. 1), samples with low EE/VS and high $\mathrm{F} / \mathrm{I}$ ratios had lower $\mathrm{pH}$ values, biogas yields and methane content, which may be explained by microbial injury due to the inhibition of LCFAs produced from the hydrolysis of lipids during the AD process, particularly in samples with a small amount of inoculum (e.g., F/I ratio higher than 0.80). In addition, the LCFA inhibition may impede the degradation of short-chain fatty acids during the hydrolysis and acidification processes (Hanaki et al., 1981; Miron et al., 2000; Palenzuela-Rollon, 1999). The low biogas yield, low $\mathrm{pH}$ and high VFA concentration during the first stage of biogas production may also be attributed to the inhibition of palmitic acid (C16:0) produced via the $\beta$-oxidation of oleic acid (C18:1) and linoleic acid (C18:2). The inhibitory effects of unsaturated LCFAs are more toxic than those of saturated LCFAs (Lalman and Bagley, 2002). For example, C16:0 may create a physical barrier on microbial cells and hinder transfer processes, thus inhibiting biogas/methane production from propionate and butyrate (Pereira et al., 2005), especially in samples with lower waste cooking oil contents and higher F/I ratios. 
However, without adjusting the $\mathrm{pH}$ values and alkalinity of the digestion system,

406

407 the $\mathrm{pH}$ recovered concomitantly as the VFA concentration decreased, which was followed by an increase in biogas production in the second stage, indicating a reversible acidification process. No significant inhibitory effect was observed when the $\mathrm{F} / \mathrm{I}$ ratios were less than 0.70 , even when the EE/VS ratios increased to $50 \%$. In addition, LCFA inhibition was reversible and could be eliminated after the depletion of biomass-associated LCFAs, namely, those with F/I ratios of 1.2, 1.0 and 0.8 . These results also indicated that the digestion process was affected by synergistic effects between the inoculum ratios and the waste cooking oil content.

\subsubsection{Ammonia nitrogen}

Digesters with higher F/I and lower EE/VS ratios exhibited higher final TAN concentrations $(p<0.01$ ), which ranged from 1345 to $1759 \mathrm{mg} / \mathrm{L}$, as well as higher TAN concentrations corresponding to higher final VFA concentrations $(p<0.01)$. The final FAN values ranged from 31.38 to $48.56 \mathrm{mg} / \mathrm{L}$, and the highest FAN value was achieved for samples with EE/VS and F/I ratios of $36 \%$ and 1.00, respectively (Table 6). The inhibitory thresholds of TAN and FAN have been reported to range from 1700 to $2500 \mathrm{mg} / \mathrm{L}$ and from 400 to $1000 \mathrm{mg} / \mathrm{L}$, respectively (Stams et al., 2003). The FAN levels were too low to inhibit the digestion process. However, it is important to note that the TAN concentrations in digesters with F/I ratios higher than 1.0 were near the reported inhibitory threshold. Additionally, an inhibition effect may appear for a FW sample with an EE/VS ratio higher than the set range and an F/I ratio within a certain range or for a $\mathrm{FW}$ sample with an $\mathrm{F} / \mathrm{I}$ ratio higher than the set range and an $\mathrm{EE} / \mathrm{VS}$ 
ratio within a certain range. Hence, in this study, the slow biogas production rate during the first stage (Fig. 1) was mainly ascribed to the acidification caused by VFAs and LCFAs. However, lower EE/VS $(p<0.01)$ and higher F/I ratios $(p<0.01)$ may lead to higher final concentrations of VFAs and TAN. Thus, higher EE/VS ratios may not necessarily lead to inhibition via the accumulation of acids (VFAs and LCFAs) or alkalis (TAN, FAN), but lower F/I ratios may do $(p<0.01)$.

\subsection{Digestate characteristics and relationships between process parameters}

The stability of the digestion process is important for maintaining sustainable anaerobic digester performance. Table 6 shows the characteristics of the final digestate including the $\mathrm{pH}$, VFA concentration, VS reduction, and protein and EE reductions at the end of digestion, which indicates the stability of the AD system. The final $\mathrm{pH}$ values (varying from 7.25 to 7.39 ) were all located in the preferred range for methanogenic activity. As one of the most important parameters for accurately controlling AD, the final VFA concentrations $(0-0.53 \mathrm{mg} / \mathrm{L})$ were very low for all of the samples at the end of the experiment, especially for samples with lower $\mathrm{F} / \mathrm{I}$ ratios and higher EE/VS ratios $(p<0.01)$, which is indicative of a complete digestion process. In the samples with lower EE/VS ratios and higher F/I ratios, the final distribution of the VFAs indicated higher concentrations of propionic and valeric acid and TAN and FAN, implying disturbances in the acetogenesis and methanogenesis pathways. Besides, only VFA $(p<0.01)$ and TAN $(p<0.01)$ were negatively correlated with EE/VS ratios $(p<0.01)$. The F/I ratios showed a significant negative correlation $(p<0.05)$ with VS 
reduction (25-43\%), whereas EE/VS failed to show any significant effect. These findings indicated that the F/I ratio had an obvious influence on VS reduction. Lower F/I and higher EE/VS ratios were for a higher EE reduction (48 - 82\%) compared with the opposite results for protein reduction (61-63\%). Previous studies reported that higher lipid content contributed to the diffusion limitations imposed by layer surrounding the bacterial cells, thus increasing the lag-phase time, and lipid hydrolysis only occurred under methanogenic conditions (Miron et al., 2000) and higher lipid content contributed to higher VFA concentrations (Li et al., 2017). Besides, lipid hydrolysis only occurred under methanogenic conditions (Miron et al., 2000). Therefore, it could be concluded higher reduction of protein correlated with a higher lipid contents. Additionally, a high lag phase time $(\lambda)$ corresponded to increased protein reduction and less VS reduction compared to no significant effect on EE reduction, which was confirmed by significant positive correlations between the $\lambda$ values and the VFA $(p<0.01)$ and TAN $(p<0.01)$ concentrations. These findings suggest that a high VFA concentration during the reversible acidification process could be conducive to protein degradation.

High $R_{m}$ values led to lower $t_{90}$ values $(p<0.01$ ), lower final concentrations of VFAs and TAN $(p<0.05)$ and shorter AD retention times $(p<0.05)$ compared to higher EE reductions $(p<0.05)$ and biogas yields $(p<0.05)$. These findings suggest that the AD efficiency of FW is significantly influenced by the content of the waste cooking and the inoculum quantity. Therefore, to achieve the maximum recoverable biogas/methane yield from $\mathrm{FW}$, it is necessary to provide organics with high 
471 biomethane potential by increasing the EE/VS ratio and to conserve a sufficient

472 amount of anaerobic microbes by starting the digestion assay at a very low F/I ratio.

473 These findings are applicable for the dilution of waste cooking oil in feedstocks with

474 higher inoculum amounts. 
475 Table 6. Final characteristics of the digestates from FW with different F/I and EE/VS ratios.

\begin{tabular}{|c|c|c|c|c|c|c|c|}
\hline EE/VS & $33 \%$ & $36 \%$ & $40 \%$ & $43 \%$ & $46 \%$ & $50 \%$ & $53 \%$ \\
\hline $\mathrm{F} / \mathrm{I}$ & 1.20 & 1.00 & 0.80 & 0.70 & 0.60 & 0.56 & 0.50 \\
\hline $\mathrm{pH}$ & $7.28 \pm 0.02$ & $7.37 \pm 0.01$ & $7.28 \pm 0.02$ & $7.39 \pm 0.16$ & $7.26 \pm 0.01$ & $7.25 \pm 0.10$ & $7.28 \pm 0.01$ \\
\hline VFA (mg/L) & $0.53 \pm 0.01$ & $0.34 \pm 0.01$ & $0.15 \pm 0.01$ & $0.38 \pm 0.00$ & $0.31 \pm 0.00$ & $0.30 \pm 0.03$ & $0.00 \pm 0.00$ \\
\hline Acetic acid (mg/L) & $0.15 \pm 0.00$ & $0.11 \pm 0.01$ & $0.11 \pm 0.01$ & $0.18 \pm 0.00$ & $0.13 \pm 0.00$ & $0.10 \pm 0.01$ & $0.00 \pm 0.00$ \\
\hline Propionic acid (mg/L) & $0.29 \pm 0.01$ & $0.14 \pm 0.00$ & $0.05 \pm 0.00$ & $0.01 \pm 0.01$ & $0.00 \pm 0.00$ & $0.01 \pm 0.01$ & $0.00 \pm 0.00$ \\
\hline Butyric acid (mg/L) & $0.00 \pm 0.00$ & $0.00 \pm 0.00$ & $0.00 \pm 0.00$ & $0.00 \pm 0.00$ & $0.00 \pm 0.00$ & $0.10 \pm 0.00$ & $0.00 \pm 0.00$ \\
\hline Valeric acid (mg/L) & $0.08 \pm 0.00$ & $0.09 \pm 0.01$ & $0.00 \pm 0.00$ & $0.08 \pm 0.00$ & $0.08 \pm 0.00$ & $0.08 \pm 0.00$ & $0.00 \pm 0.00$ \\
\hline TAN (mg/L) & $1759 \pm 26$ & $1665 \pm 20$ & $1567 \pm 25$ & $1446 \pm 18$ & $1501 \pm 17$ & $1408 \pm 21$ & $1345 \pm 49$ \\
\hline FAN (mg/L) & $41.94 \pm 6.75$ & $48.56 \pm 4.82$ & $37.35 \pm 5.90$ & $44.12 \pm 6.89$ & $34.21 \pm 9.18$ & $31.38 \pm 7.12$ & $32.07 \pm 4.89$ \\
\hline VS reduction $(\%)$ & 25 & 28 & 42 & 43 & 42 & 42 & 39 \\
\hline Protein reduction $(\%)$ & 63 & 63 & 62 & 62 & 62 & 61 & 61 \\
\hline EE reduction $(\%)$ & 48 & 56 & 61 & 60 & 67 & 82 & 86 \\
\hline
\end{tabular}




\subsection{Relationships between responses and independent variables}

These findings suggested that both the substrate composition and the inoculation ratio co-affect the digestion parameters during $\mathrm{AD}$. The EE/VS and F/I ratios were selected as independent variables, and nine corresponding parameters including organics reduction and performance stability parameters (VS, protein and EE reduction; methane content; $t_{90}$; VFA and TAN final concentrations; and kinetic parameters) were selected as the dependent variables. The coefficients of the second-order polynomial models (Eq. (2)) corresponding to each dependent variable were evaluated and are listed in Table 7 . The $R^{2}$ values for these models ranged from 0.839 to 0.999 , indicating that the data can be well explained by these models, as the $R^{2}$ values are all greater than 0.75 (Naik and Setty, 2014). Additionally, in some cases, the terms $(E E / V S) \times(F / I)$ and $(E E / V S)^{2}$ were removed from the final polynomial to achieve lower $p$ values (their coefficients were set to zero). All of the $p$ values were lower than 0.05 , so we can concluded that the model term was significant.

$$
\text { For FW digestion in practice, high biogas/methane production with a short }
$$
retention time is always preferred as long as sustainable digestion is guaranteed.

Considering the relationships between the performance and kinetic parameters and the $\mathrm{F} / \mathrm{I}$ and EE/VS ratios, optimizing the F/I and EE/VS ratios to achieve high methane yield and biogas production efficiency without inhibition is necessary. The results of the second-order polynomials in terms of the F/I and EE/VS ratios in this study may also be used as a reference for experimental batch AD of FW to predict the system stability and to avoid inhibition (Table 7). 
Table 7. Coefficients from the regression models.

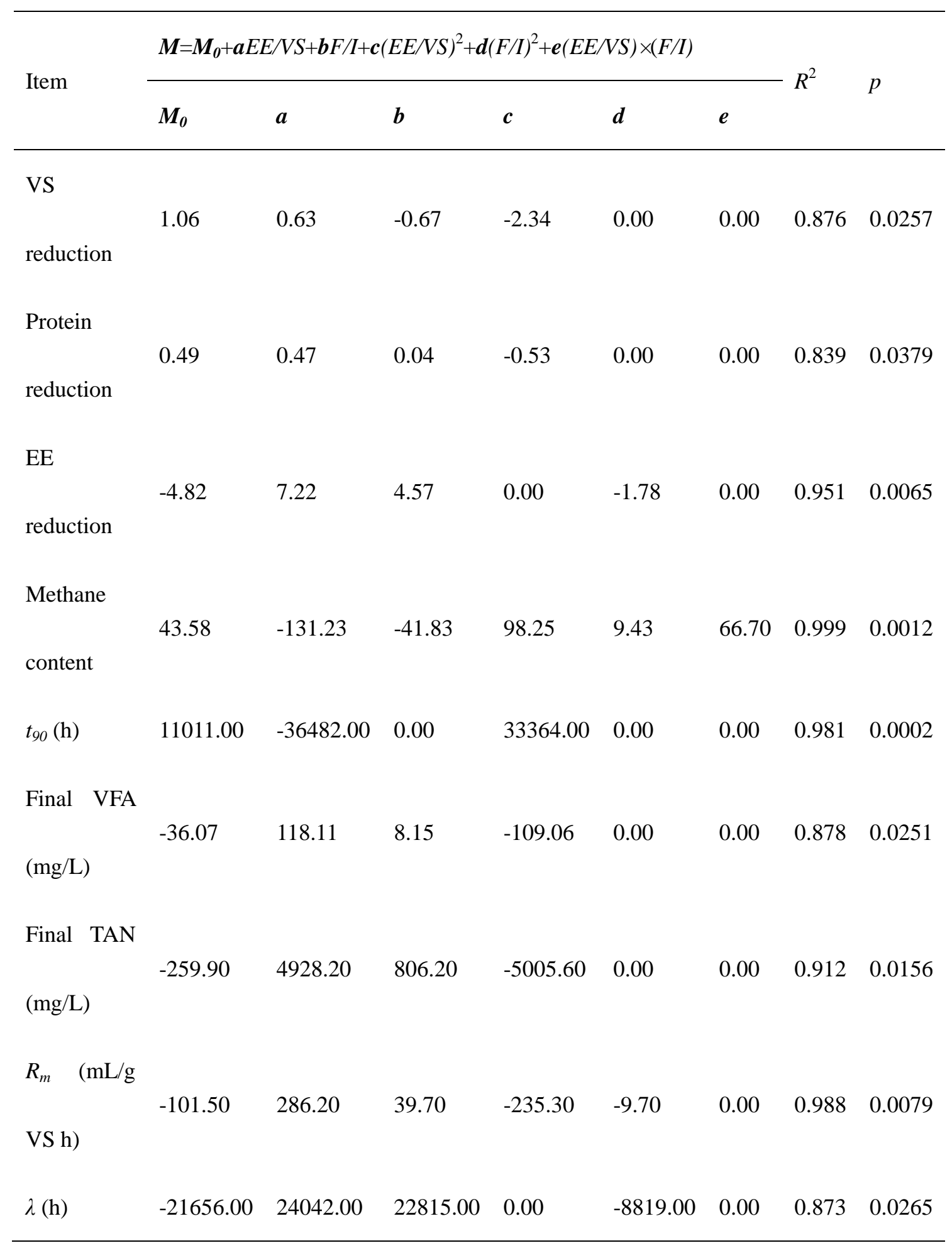

499 In addition, some problems associated with process instability can occur when the 500 results from the BMP tests are applied in practice, as it is much easier to control their 501 digestion parameters (e.g., feedstock composition, temperature, and anaerobic 
502

503

504

505

506

507

508

509

510

511

512

513

514

515

516

517

518

519

520

521

522

523

524

environment) compared to pilot projects. A higher F/I ratio is always preferred due to cost and space savings, whereas a relatively larger EE/VS ratio is good for obtaining higher methane yield from a FW AD system. Therefore, to achieve the highest possible biogas/methane yield, a low F/I ratio (such as $0.5-0.7$ ) combined with an ideal EE/VS ratio of slightly less than $43 \%$ is preferred.

\section{Conclusions}

For EE/VS ratios lower than 40\%, F/I ratios higher than 0.8 resulted in reversible acidification and possible LCFA inhibition along with lower biogas/methane yields and a longer lag phase. To minimize the possible inhibition caused by high EE/VS ratios during $\mathrm{FW}$ digestion, the $\mathrm{F} / \mathrm{I}$ ratio should be lower than 0.70 , which enabled the maintenance of a high biogas conversion ratio (82-94\%) with high organics reduction and a short lag time (267-430 h). The optimum EE/VS and F/I ratios for the AD of FW are $43 \%$ and 0.70 , respectively, as they resulted in the highest biogas yield and methane content and the largest VS reduction.

\section{Acknowledgements}

This work was supported financially by the Major Science and Technology Program for Water Pollution Control and Treatment (2017ZX07202005) and the China Scholarship Council (CSC).

\section{E-supplementary data for this work can be found in e-version of this paper online} (Table A1). 
Alves, M.M., Pereira, M.A., Sousa, D.Z., Cavaleiro, A.J., Picavet, M., Smidt, H., Stams, A.J.M., 2009. Waste lipids to energy: how to optimize methane production from long-chain fatty acids (LCFA). MICROB BIOTECHNOL 2, 538-550.

Angelidaki, I., Sanders, W., 2004. Assessment of the anaerobic biodegradability of macropollutants. Reviews in Environmental Science and Biotechnology 3, 117-129.

APHA, 1992. Standard Methods for the Examination of Water and Wastewater, 18th ed. American Public Health Association, Washington, DC.

Boulanger, A., Pinet, E., Bouix, M., Bouchez, T., Mansour, A.A., 2012. Effect of inoculum to substrate ratio (I/S) on municipal solid waste anaerobic degradation kinetics and potential. WASTE MANAGE 32, 2258-2265.

Chen, J.L., Ortiz, R., Steele, T.W.J., Stuckey, D.C., 2014. Toxicants inhibiting anaerobic digestion: A review. BIOTECHNOL ADV 32, 1523-1534.

Chen, Y., Cheng, J.J., Creamer, K.S., 2008. Inhibition of anaerobic digestion process: a review. BIORESOURCE TECHNOL 99, 4044-4064.

Cirne, D.G., Paloumet, X., Bj Rnsson, L., Alves, M.M., Mattiasson, B., 2007. Anaerobic digestion of lipid-rich waste - Effects of lipid concentration. RENEW ENERG 32, 965-975.

Dechrugsa, S., Kantachote, D., Chaiprapat, S., 2013. Effects of inoculum to substrate ratio, substrate mix ratio and inoculum source on batch co-digestion of grass and pig manure. BIORESOURCE TECHNOL 146, 101-108.

Fagbohungbe, M.O., Herbert, B.M.J., Li, H., Ricketts, L., Semple, K.T., 2015. The effect of substrate to inoculum ratios on the anaerobic digestion of human faecal material. Environmental Technology \& Innovation 3, 121-129.

Fernández, J., Pérez, M., Romero, L.I., 2008. Effect of substrate concentration on dry mesophilic anaerobic digestion of organic fraction of municipal solid waste (OFMSW). BIORESOURCE TECHNOL 99, 6075-6080.

González-Fernández, C., García-Encina, P.A., 2009. Impact of substrate to inoculum ratio in anaerobic digestion of swine slurry. Biomass and Bioenergy 33, 1065-1069.

Haider, M.R., Zeshan, Yousaf, S., Malik, R.N., Visvanathan, C., 2015. Effect of mixing ratio of food waste and rice husk co-digestion and substrate to inoculum ratio on biogas production. BIORESOURCE TECHNOL 190, 451-457.

Hanaki, H., Matsuo, T., Nagase, M., 1981. Mechanism of inhibition caused by long chain fatty acids in anaerobic digestion processes. BIOTECHNOL BIOENG 7, 1591-1610.

Hwu, C., Donlon, B., Lettinga, G., 1996. Comparative toxicity of long-chain fatty acid to anaerobic sludges from various origins. WATER SCI TECHNOL 34, 351-358.

Kawai, M., Nagao, N., Tajima, N., Niwa, C., Matsuyama, T., Toda, T., 2014. The effect of the labile organic fraction in food waste and the substrate/inoculum ratio on anaerobic digestion for a reliable methane yield. BIORESOURCE TECHNOL 157, 174-180.

Koch, K., Helmreich, B., Drewes, J.R.E., 2015. Co-digestion of food waste in municipal wastewater treatment plants: Effect of different mixtures on methane yield and hydrolysis rate constant. APPL ENERG 137, 250-255.

Labatut, R.A., Angenent, L.T., Scott, N.R., 2011. Biochemical methane potential and biodegradability of complex organic substrates. BIORESOURCE TECHNOL 102, 2255-2264.

Lalman, J., Bagley, D.M., 2002. Effects of C18 long chain fatty acids on glucose, butyrate and hydrogen degradation. WATER RES 36, 3307-3313. 
Lesteur, M., Bellon-Maurel, V., Gonzalez, C., Latrille, E., Roger, J.M., Junqua, G., Steyer, J.P., 2010. Alternative methods for determining anaerobic biodegradability: a review. PROCESS BIOCHEM 45, 431-440.

Li, Y., Jin, Y., Borrion, A., Li, H., Li, J., 2017. Effects of organic composition on mesophilic anaerobic digestion of food waste. BIORESOURCE TECHNOL 244, 213-224.

Li, Y., Jin, Y., Li, J., 2016. Influence of thermal hydrolysis on composition characteristics of fatty acids in kitchen waste. ENERGY 102, 139-147.

Li, Y., Jin, Y., Li, J., Li, H., Yu, Z., 2016. Effects of thermal pretreatment on the biomethane yield and hydrolysis rate of kitchen waste. APPL ENERG 172, 47-58.

Liu, G., Zhang, R., El-Mashad, H.M., Dong, R., 2009. Effect of feed to inoculum ratios on biogas yields of food and green wastes. BIORESOURCE TECHNOL 100, 5103-5108.

Long, J.H., Aziz, T.N., Francis, L., Ducoste, J.J., 2012. Anaerobic co-digestion of fat, oil, and grease (FOG): a review of gas production and process limitations. PROCESS SAF ENVIRON 90, 231-245.

Mata-Alvarez, J., Mace, S., Llabres, P., 2000. Anaerobic digestion of organic solid wastes. An overview of research achievements and perspectives. BIORESOURCE TECHNOL 74, 3-16.

Miron, Y., Zeeman, G., van Lier, J.B., Lettinga, G., 2000. The role of sludge retention time in the hydrolysis and acidification of lipids, carbohydrates and proteins during digestion of primary sludge in CSTR systems. WATER RES 34, 1705-1713.

Moset, V., Al-zohairi, N., M Ller, H.B., 2015. The impact of inoculum source, inoculum to substrate ratio and sample preservation on methane potential from different substrates. Biomass and Bioenergy 83, 474-482.

Naik, S.S., Setty, Y.P., 2014. Optimization of parameters using response surface methodology and genetic algorithm for biological denitrification of wastewater. INT J ENVIRON SCI TE 11, 823-830.

Naumann, C., Bassler, R., 1993. Methodenbuch Band III. Die chemische Untersuchung von Futtermitteln. Darmstadt, Germany: VDLUFA-Press.

Nie, Y., Jin, Y., Liu, F., 2013. Handbook on solid waste management and technology. Chemical Industry Press.

Niu, Q., Hojo, T., Qiao, W., Qiang, H., Li, Y., 2014. Characterization of methanogenesis, acidogenesis and hydrolysis in thermophilic methane fermentation of chicken manure. CHEM ENG J 244, 587-596. Palatsi, J., Laureni, M., Andrés, M.V., Flotats, X., Nielsen, H.B., Angelidaki, I., 2009. Strategies for recovering inhibition caused by long chain fatty acids on anaerobic thermophilic biogas reactors. BIORESOURCE TECHNOL 100, 4588-4596.

Palenzuela-Rollon, A., 1999. Anaerobic digestion of fish wastewater with special emphasis on hydrolysis of suspended solids. Ph.D. thesis. Agricultural University, Wageningen.

Pellera, F., Gidarakos, E., 2016. Effect of substrate to inoculum ratio and inoculum type on the biochemical methane potential of solid agroindustrial waste. Journal of Environmental Chemical Engineering 4, 3217-3229.

Pereira, M.A., Pires, O.C., Mota, M., Alves, M.M., 2005. Anaerobic biodegradation of oleic and palmitic acids: Evidence of mass transfer limitations caused by long chain fatty acid accumulation onto the anaerobic sludge. BIOTECHNOL BIOENG 92, 15-23.

Raposo, F., Banks, C.J., Siegert, I., Heaven, S., Borja, R., 2006. Influence of inoculum to substrate ratio on the biochemical methane potential of maize in batch tests. PROCESS BIOCHEM 41, 1444-1450.

Ren, L., Nie, Y., Liu, J., 2013. Influencing factors of biodiesel production from waste cooking oil. Acta 
Scientiae Circumstantiae 33, 1104-1109.

Siles, J.A., Brekelmans, J., Martin, M.A., Chica, A.F., Martin, A., 2010. Impact of ammonia and sulphate concentration on thermophilic anaerobic digestion. BIORESOURCE TECHNOL 101, 9040-9048.

Stams, A., Elferink, S.O., Westermann, P., 2003. Metabolic interactions between methanogenic consortia and anaerobic respiring bacteria. Springer, pp. 31-56.

Sun, Y., Wang, D., Yan, J., Qiao, W., Wang, W., Zhu, T., 2014. Effects of lipid concentration on anaerobic co-digestion of municipal biomass wastes. WASTE MANAGE 34, 1025-1034.

Tang, Y., Koike, Y., Liu, K., An, M., Morimura, S., Wu, X., Kida, K., 2008. Ethanol production from kitchen waste using the flocculating yeast Saccharomyces cerevisiae strain KF-7. Biomass and Bioenergy 32, 1037-1045.

Wang, Y., Zhang, Y., Wang, Z., Meng, L., 2009. Effects of volatile fatty acid concentrations on methane yield and methanogenic bacteria. BIOMASS BIOENERG 33, 848-853.

Zhang, B., Zhang, L.L., Zhang, S.C., Shi, H.Z., Cai, W.M., 2005. The influence of pH on hydrolysis and acidogenesis of kitchen wastes in two-phase anaerobic digestion. ENVIRON TECHNOL 26, 329-339.

Zhang, C., Su, H., Baeyens, J., Tan, T., 2014. Reviewing the anaerobic digestion of food waste for biogas production. Renewable and Sustainable Energy Reviews 38, 383-392.

Zhang, W., Lang, Q., Fang, M., Li, X., Bah, H., Dong, H., Dong, R., 2017. Combined effect of crude fat content and initial substrate concentration on batch anaerobic digestion characteristics of food waste. BIORESOURCE TECHNOL 232, 304-312.

Zhang, Y., Dube, M.A., McLean, D.D., Kates, M., 2003. Biodiesel production from waste cooking oil: 2. Economic assessment and sensitivity analysis. BIORESOURCE TECHNOL 90, 229-240.

Zhao, C., Cui, X., Liu, Y., Zhang, R., He, Y., Wang, W., Chen, C., Liu, G., 2017. Maximization of the methane production from durian shell during anaerobic digestion. BIORESOURCE TECHNOL 238, 433-438.

Zhuang, J., Zhiqiang, F., Chunrong, L., Dan, L., Fangmei, Z., Zhongyang, X., Quanfeng, L., Yupeng, T., 2013. A food waste cooking oil identification methods, 2014/10/29. 